\title{
Symptom Dimensions as Predictors of Clinical Outcome, Duration of Hospitalization, and Aggressive Behaviours in Acutely Hospitalized Patients with Psychotic Exacerbation
}

\author{
Alessandro Colasanti ${ }^{1,2}$, Silvia Paletta ${ }^{1}$, Donatella Moliterno ${ }^{1}$, Alessandra Mazzocchi ${ }^{1}$, Massimo \\ Carlo Mauri ${ }^{1, *}$ and Alfredo Carlo Altamura ${ }^{1}$ \\ ${ }^{I}$ Clinical Psychiatry, Clinical Neuropsychopharmacology Unit, University of Milan, Fondazione IRCCS Ca' Granda, \\ Ospedale Maggiore Policlinico, Via F.Sforza 35, 20122 Milan, Italy \\ ${ }^{2}$ Neuropsychopharmacology Unit, Division of Experimental Medicine, Imperial College, Hammersmith Hospital, 160 \\ Du Cane Road, W129LL, London, UK
}

\begin{abstract}
In the present study we extract clusters of symptoms in acute hospitalized psychotic patients during a reexacerbation phase, using factor analysis of BPRS-E. We aim to investigate the relative contribution of each symptom dimension in predicting the severity of symptoms at discharge, the length of acute hospitalization, and the occurrence of aggressive behaviours during acute hospitalization. The data are drawn from a prospective, naturalistic, observational study of 183 patients with Psychotic Disorders consecutively admitted to a psychiatric ward, during a re-exacerbation phase. General symptomatology has been measured through BPRS-E at admission and at discharge. Statistical analyses include principal component analysis and multiple linear regression.

We found symptoms of acute psychosis disorder to cluster together in four distinct domains, labelled "Excitement/Activation", "Positive symptoms", and "Negative symptoms", and "Depression/Anxiety".

Excitement/activation was the dimension most associated with occurrence of aggressive behaviours and severity of psychopathological symptoms at discharge. The negative symptoms dimension, also, predicted the severity of symptoms at discharge. Positive and negative symptoms dimensions were both predictors of duration of hospitalization. The depressive dimension was significantly associated only to self-aggression. These data indicate that during acute hospitalization due to re-exacerbation of psychosis each symptom dimension has a specific impact on distinct measures of outcome.
\end{abstract}

Keywords: Acute psychosis, schizophrenia, excitement, activation, hyperactivity, mania, positive and negative symptoms, depression, factor analysis, symptom clusters, duration of hospitalization, aggression, violence, inpatients.

\section{INTRODUCTION}

A large amount of data from literature suggests that a dimensional approach to symptoms domains in schizophrenia is valuable not only in describing clinical picture, but also in predicting outcome in clinical practice, also in the very short term such as during acute hospitalization. The present paper aims to replicate and extend previous investigations on this topic, by testing the hypothesis that in acute psychotic patients during re-exacerbation phase distinct symptom dimensions can differentially predict practical measures of poor clinical outcome during acute hospitalization.

Studies applying principal component analysis (PCA) on Brief Psychiatric Rating Scale (BPRS) [1] indicated that symptoms on schizophrenia cluster together in distinct domains. A four factors solution resulted as the most interpretable [2,3]. Dimensions are often defined as excite-

\footnotetext{
*Address correspondence to this author at the Clinical Neuropsychopharmacology Unit, University of Milan, IRCCS Ospedale Maggiore Policlinico, Via F.Sforza 35, 20122 Milan, Italy; Tel: 0039 0255035997; Fax: 0039 0255035990; E-mail: maurimc@policlinico.mi.it
}

ment/activation, positive symptoms, negative symptoms, and depression. A large body of research was conducted on BPRS of 18 items, with only few factor analysis studies performed on the expanded version including 24 items (BPRSE) [2, 4-9]. The BPRS-E includes six extra items (suicidality, elevated mood, bizarre behaviour, self-neglect, distractibility and motor hyperactivity) and has been designed in order to increase the coverage of the instrument. It has defined scale points and probe questions in order to increase its interrater reliability [10]. Research pertaining to the dimensional structure of the BPRS-E have assessed different populations and used different techniques, mainly based on PCA or factor analysis approach. Most of the studies confirmed the consistency of the 4-factors solution $[2,4,11]$.

In the present study, we focus on three dependent variables to investigate the relative contribution of each symptom dimension in predicting different aspects of short - term clinical outcome in clinical practice: the severity of symptoms at discharge, the length of acute hospitalization, and the presence of aggressive or self-aggressive behaviours during acute hospitalization. Each of these are practical outcome measures that significantly affect the clinical management of acute psychotic patients in the short term. 
First, we want to investigate which one of the four factors commonly extracted in factor analyses on BPRS (depression/anxiety; positive symptoms; negative symptoms; excitement/activation) is the best correlate of the severity of residual symptoms at discharge after acute treatment. A number of reports have suggested that general psychopathology at admission and either severe positive or negative symptoms [12-15] predict a poor outcome in the short-term. Other studies found that among other clinical and sociodemographic factors, exclusively the severity of positive symptoms predicted a better response to treatment in acute exacerbations [16]. Moreover, it has been also suggested that depressive symptoms at baseline in an acute episode of schizophrenia are prognostic of a more favourable treatment response during short-term treatment $[10,11]$.

The duration of acute hospitalization could be also considered an indirect indicator of poor outcome. Studies applying factor analysis and regression analysis of BPRS-E symptoms in hospitalized psychiatric patients with persistent mental illness found that the severity of negative symptoms was the best predictor of the inpatient length of stay [18]. These studies also included patients other than schizophrenic but analysed the clinical outcome in the medium-long term.

Aggression committed by acutely hospitalized patients with acute psychosis is also highly relevant from a clinical point of view, as it poses a significant psychiatric service problem. Many investigators have found psychiatric symptoms to contribute to aggressive behavior in hospitalized psychiatric patients [19] and measures of aggression have been found to be significantly associated with both positive symptoms and negative symptoms of schizophrenia [20, 21].

A number of studies have also showed that in early-phase psychotic disorders the symptoms found to be positively associated with aggression are those typically found in mania, for instance overactivation, expansive mood, or emotional turmoil [22-24].

In summary, with the present study we aim to examine symptoms' dimensions in acutely hospitalized patients with psychotic re-exacerbation and compare the relative importance of each dimension in predicting measures of poor clinical outcome, such as persistence of severe psychopathology at discharge, prolonged stay in the acute ward, and occurrence of aggressive behaviours during hospitalization.

\section{MATERIALS AND METHODS}

The data were drawn from a prospective, naturalistic, observational study of patients with Psychotic Disorders during an exacerbation phase of psychosis, aged 19-80 years, acutely admitted to the Servizio Psichiatrico di Diagnosi e Cura (SPDC), Psychiatric Clinic, IRCCS Foundation Ospedale Maggiore Policlinico of Milan, Italy, between January 2006 and March 2007. During the study period, all the patients with a diagnosis of psychotic disorder in exacerbation phase at presentation were consecutively enrolled in the study as soon as they were admitted in the ward. Two days of hospitalisation at least were required to include the patients in the study. No patient, who satisfied these criteria, has been excluded. The SPDC is an in-patient psychiatric service that offers community-based mental health emergency care. According to the local organization of the mental health, the urban area of Milan is divided into zones, each of these providing an acute inpatient psychiatric facility for the population of reference. Therefore, this SPDC is the only acute in-patient ward in the area and the only acute psychiatric service offering emergency care to an urban population of approximately 190,000 people living in the centre of Milan. This SPDC was composed by 24 beds, 5 experienced physicians, 4 residents, 16 nurses. The service provides emergency and acute cares, and the mean duration of hospitalisation was about 12 days.

The protocol received agreement by a local Ethics Committee and the patients or their relatives provided their written informed consent.

Diagnoses were performed by multiple expert clinicians according to DSM IV TR axis I criteria. The inter-rater reliability was above $90 \%$.

Eligible participants completed the BPRS-E at admission (T0) and at discharge (T1) according to the psychopathological status of the patient at this time. Well-trained examiners, who were blinded to the diagnosis, pursued all the assessments. In order to obtain inter-rater consistency in their assessments, the examiners were trained about the use of BPRS-E before starting the administration.

BPRS-E is a semi-structured 24-items interview that rates general psychopathology on a Liker scale from 0 (absent) to 7 (extremely severe). Anchor points and a manual are provided for scoring discernment [10]. Symptoms measured by the BPRS-E are somatic concernes, anxiety, depression, suicidality, guilt, hostility, elevated mood, grandiosity, suspiciousness, hallucinations, unusual thought contents, bizarre behaviour, self-neglect, disorientation, conceptual disorganization, emotional withdrawal, motor retardation, tension, uncooperativeness, excitement, distractibility, motor hyperactivity, mannerisms and posturing.

We used the Modified Overt Aggression Scale (MOAS) [25] to assess aggressive and violent behaviours occurred during the hospitalization. MOAS scores were attributed by a single researcher blind to clinical diagnosis, in accord with the information given from nurses, doctors, relatives, patients, and other significants.

MOAS is a semi-structured interview that value four clusters of aggressive behaviour: verbal aggression, aggression against objects, self- aggression, aggression against others. Every subscale scores ranged between 0 (no aggression) and 4 (maximal score). The subscales were weighted as described by Kay et al. [25]: we attributed to each cluster a weight from 1 (minimum weight) to 4 (maximum weight), in order to minimize the effect of verbal aggression compared to other more severe types of aggression. Thus each score was multiplied for a pre-defined coefficient, respectively 1 for verbal aggression, 2 for aggression against objects, 3 for self- aggression, 4 for aggression against others. The sum of the weighted scores represents the MOAS total score.

The statistical analyses were performed by SPSS 17.0.

Spearman's correlation analysis was performed between BPRS scores at $\mathrm{T} 0$ and $\mathrm{T} 1$, and between BPRS scores or MOAS scores, and duration of hospitalisation. Nonparametric correlation was elected because of the expected 
Table 1. Clinical and Demographic Characteristics of Patients

\begin{tabular}{|c|c|}
\hline Variable & Mean (SD) \\
\hline Age (years) & $42.2(11.2)$ \\
\hline Education (years) & $10.4(3.6)$ \\
\hline BPRS total score at admission & $55.3(13.9)$ \\
\hline BPRS total score at discharge & $39.2(9.8)$ \\
\hline Duration of hospitalization (days) & $12.0(8.6)$ \\
\hline & $\mathbf{n}(\%)$ \\
\hline Males & $146(79.8 \%)$ \\
\hline Unemployed & $117(63.9 \%)$ \\
\hline Married & $23(12.6 \%)$ \\
\hline Drug abusers & $49(26.8 \%)$ \\
\hline Alcohol abusers & $43(23.5 \%)$ \\
\hline Aggressive before admission & $80(43.7 \%)$ \\
\hline Involuntary status at admission & $55(30.1 \%)$ \\
\hline
\end{tabular}

non-normal, skewed distribution of BPRS and MOAS scores and presence of outliers. Spearman's coefficient also has some advantages over Pearson's coefficient when analyzing psychiatric rating scales in terms of higher specificity despite negligible lower sensitivity [26].

Principal Component analysis with Varimax rotation of BPRS-E items was performed to obtain variables with very high factors weights in some factors and very low factor weights in others. The Principal Component Factor analysis was applied only on the BPRS symptoms at baseline. To insert a variable in a factor, a factor weight of at least 0.4 was selected as the threshold value. Factor Scores were then computed as variables, representing a measure of each subject's contribution to each factor. Then, Linear Regression analyses were executed to evidence the relative weight of each Principal Component in the determination of the dependent variables indicating clinical outcome. These dependent variables were BPRS scores at T1, the duration of acute hospitalisation, and MOAS total and subscale scores. The predicting variables were the Principal Factor scores obtained by factor analysis and have been entered in the model in a single step.

\section{RESULTS}

183 patients $(140 \mathrm{M}, 43 \mathrm{~F})$ with an acute psychotic disorder were included in the study. The mean age was 42.2 years \pm 11.2 SD. The mean duration of hospitalization was 12 days \pm 8.6 SD. Each of the 183 patients was observed until the end of the hospitalization. Socio-demographic and clinical characteristics of patients are listed in Table $\mathbf{1}$.

106 patients received a diagnosis of Schizophrenia, Schizoaffective or Schizophreniform disorder, Other diagnoses were Delusional disorder $(n=12)$, Brief Psychotic Disorder $(n=8)$ Substance-Induced Psychotic Disorders $(n=35)$, Psychotic Disorder due to general medical condition $(n=3)$. and Psychotic Disorder not otherwise specified $(n=19)$.
The total BPRS score showed a significant improvement at $\mathrm{T} 1$ compared to $\mathrm{T} 0(39.21 \pm 9.78 \mathrm{SD}$ vs $55.34 \pm 13.94 \mathrm{SD}$, $\mathrm{t}=16.7, \mathrm{df}=182, \mathrm{p}<0.0001)$.

Aggressive behaviours occurred in 20 cases (10.9\% of the cases). Verbal aggression occurred in 17 cases $(9.3 \%)$, aggression against objects in 3 cases (1.6\%), self- aggression in 2 cases $(1.1 \%)$, and aggression against others occurred in 7 cases $(3.8 \%)$.

The duration of hospitalization was positively correlated to the BPRS T0 scores but there was no correlation between duration of hospitalization and BPRS T1 scores. No correlation was found between duration of hospitalization and MOAS total and subscale scores, except for self-aggression score $(\mathrm{r}=0.178 ; \mathrm{p}<0.05)$.

As shown in Table 2 , the factor analysis produced a model with four factors, respectively labelled Excitement (Activation); Positive symptoms (Resistance); Negative symptoms; Depression (Anxiety).

Twenty-two items loaded on the factors, which accounted for $48.6 \%$ of variance.

Each cluster was labelled according to the most represented symptoms; only factor weight $>0.4$ were taken into consideration.

The 4 components were: the EXCITEMENT/ACTIVATION dimension consisting in excitement, elevated mood, motor hyperactivity, bizarre behaviour, grandiosity; tension; mannerism and posturing. The POSITIVE SYMPTOMS dimension included uncooperativeness, suspiciousness, hostility, and unusual thought content; the NEGATIVE SYMPTOMS dimension was characterized by blunted affect, conceptual disorganization, self-neglect, distractibility, disorientation, motor retardation, and emotional withdrawal. The DEPRESSION/ANXIETY dimension consisted in depression, anxiety, suicidality, and somatic concern. Guilt and hallucinations had low weight scores in each component, the highest being 0.37 and 0.29 respectively, both loading on the depression/anxiety component. The factor extracted explained $48.6 \%$ of the variance.

The regression analyses conducted on the four factors extracted by Factor Analysis as predictors of outcome measures are shown in Tables 3-5.

The strongest predictors of BPRS score at discharge were the EXCITEMENT/ACTIVATION factor $(\beta=0.31$; $p<0.0001)$ and the NEGATIVE SYMPTOMS factor $(\beta=$ $0.29 ; p<0.0001)$. The POSITIVE SYMPTOMS and the DEPRESSION/ANXIETY factor showed low and nonsignificant $\beta$-coefficients (Table $\mathbf{3}$ ).

The most important predictor of duration of hospitalization was the POSITIVE SYMPTOMS factor $(\beta=0.22$; $\mathrm{p}<0.005)$. The NEGATIVE SYMPTOMS factor was also a significant predictor but the strength of correlation was relatively weak $(\beta=0.16 ; p<0.05)$. The EXCITEMENT/ACTIVATION factor and the DEPRESSION/ANXIETY factor were non-significant (Table 4).

The relative contribute of symptom factors in predicting MOAS total and subscale scores is showed in Table 5. The 
Table 2. Principal Component Analysis of BPRS-E Symptoms. Factors Extracted: Activation/Excitement, Resistance/Positive symptoms, Depression/Anxiety, and Negative symptoms. Only factor weights $>0.4$ are showed in the table. Guilt and Hallucination are not Present Because their Factor Weight was Below 0.4 in Every Component

\begin{tabular}{|c|c|c|c|c|}
\hline BPRS Symptom & $\begin{array}{l}\text { Activation / } \\
\text { Excitement }\end{array}$ & $\begin{array}{c}\text { Resistance / } \\
\text { Positive S. }\end{array}$ & Negative $\mathbf{S}$. & $\begin{array}{c}\text { Depression / } \\
\text { Anxiety }\end{array}$ \\
\hline excitement & 0.80 & & & \\
\hline elevated mood & 0.77 & & & \\
\hline motor hyperactivity & 0.73 & & & \\
\hline bizzarre behaviour & 0.63 & & & \\
\hline grandiosity & 0.63 & & & \\
\hline tension & 0.55 & & & \\
\hline mannerism and posturing & 0.54 & & & \\
\hline cooperativeness & & 0.80 & & \\
\hline suspiciousness & & 0.76 & & \\
\hline hostility & & 0.74 & & \\
\hline unusual thought content & & 0.45 & & \\
\hline blunted affect & & & 0.62 & \\
\hline conceptual disorganization & 0.40 & & 0.61 & \\
\hline self-neglect & & & 0.59 & \\
\hline distractibility & 0.42 & & 0.58 & \\
\hline disorientation & & & 0.53 & \\
\hline motor retardation & & & 0.50 & \\
\hline emotional withdrawal & & 0.44 & 0.48 & \\
\hline depression & & & & 0.74 \\
\hline anxiety & & & & 0.73 \\
\hline suicidality & & & & 0.55 \\
\hline somatic concern & & & & 0.53 \\
\hline
\end{tabular}

Table 3. Linear Regression. Dependent Variable: Total BPRS-E Score at Discharge. Predictors: Factor Scores of Baseline BPRS-E components'; * p <0.05

BPRS Score at Discharge

\begin{tabular}{|c|c|c|c|c|c|c|c|}
\hline COMPONENT & B & $\mathbf{S E}$ & $\beta$ & $\mathbf{T}$ & $\mathbf{p}$ & \multicolumn{2}{|c|}{$95 \% \mathrm{CI}$} \\
\hline Activation/Excitement & 2.99 & 0.65 & 0.31 & 4.58 & $<0.0001^{*}$ & 1.70 & 4.28 \\
\hline Negative $S$. & 2.86 & 0.65 & 0.29 & 4.38 & $<0.0001^{*}$ & 1.57 & 4.15 \\
\hline Depression/Anxiety & 1.12 & 0.65 & 0.12 & 1.72 & 0.09 & -0.17 & 2.41 \\
\hline
\end{tabular}

only significant observed predictor of total MOAS scores was the EXCITEMENT/ACTIVATION factor $(\beta=0.21$; $\mathrm{p}<0.01)$, which also significantly predicted aggression against others scores $(\beta=0.16 ; \mathrm{p}<0.05)$. Verbal aggression was significantly predicted by the EXCITEMENT/ACTIVATION and POSITIVE SYMPTOMS factors $(\beta=0.25 ; p=0.01$ and $\beta=0.15 ; p<0.05$, respectively). The DEPRESSION/ANXIETY factor significantly predicted MOAS self-aggression scores $(\beta=0.24 ; p=0.001)$.

\section{DISCUSSION}

In the present study we have extracted clusters of symptoms in schizophrenic-spectrum patients acutely admitted to a psychiatric emergency facility, by using factor analysis of BPRS-E. Subsequently, we have investigated which of the extracted symptom dimensions best predicted the severity of symptoms at discharge, the length of acute hospitalization, and the occurrence of different subtype of aggressive behaviours during the stay in an acute psychiatric ward. 
Table 4. Linear Regression. Dependent variable: duration of hospitalization. Predictors: factor scores of baseline BPRS-E components'; * $p<0.05$

Duration of Hospitalization

\begin{tabular}{|c|c|c|c|c|c|c|c|}
\hline COMPONENT & $\mathbf{B}$ & $\mathbf{S E}$ & $\boldsymbol{\beta}$ & $\mathbf{T}$ & \multicolumn{2}{|c|}{$\mathbf{p}$} & \multicolumn{2}{|c|}{$\mathbf{9 5 \%} \mathbf{C I}$} \\
\hline \hline Activation/Excitement & 0.77 & 0.62 & 0.09 & 1.25 & 0.21 & -0.44 & 0.77 \\
\hline Resistance/Positive s. & 1.86 & 0.62 & 0.22 & 3.02 & $<0.005^{*}$ & 0.65 & 1.86 \\
\hline Negative S. & 1.33 & 0.62 & 0.16 & 2.17 & $0.03^{*}$ & 0.12 & 1.33 \\
\hline Depression/Anxiety & 1.03 & 0.62 & 0.12 & 1.68 & 0.09 & -0.18 & 1.03 \\
\hline
\end{tabular}

Table 5. Linear Regression. Dependent variable: total MOAS score. Predictors: Factor Scores of Baseline BPRS-E Components'; * p $<0.05$

MOAS Total Score

\begin{tabular}{|c|c|c|c|c|c|c|c|}
\hline COMPONENT & B & SE & $\beta$ & $\mathbf{T}$ & $\mathbf{p}$ & \multicolumn{2}{|c|}{$95 \% \mathrm{CI}$} \\
\hline Activation/Excitement & 0.44 & 0.16 & 0.21 & 2.83 & $\mathrm{P}<0.01 *$ & 0.13 & 0.76 \\
\hline Negative $S$. & 0.04 & 0.16 & 0.02 & 0.24 & 0.81 & -0.27 & 0.35 \\
\hline Depression/Anxiety & 0.29 & 0.16 & 0.13 & 1.82 & 0.07 & -0.02 & 0.60 \\
\hline
\end{tabular}

The first aim of the study was to identify symptoms' dimensions in acute schizophrenic patients. As expected, the factor analysis of the present study on acute schizophrenic patients found 4 major dimensions:, "Excitement/Activation", "Positive symptoms", "Negative symptoms", and "Depression/Anxiety" (Table 2). The four factors identified are similar, but not identical, to other factor solutions proposed for the BPRS. In most previous studies some components were almost constantly present: positive symptoms, negative symptoms, depression or affect $[3,7,17,18$, $25,27]$. A study which reported factor analyses of BPRS-E in a selected sample of chronic schizophrenic patients found four dimension labelled positive symptoms, negative symptoms, depression/anxiety, agitation/mania, similarly to our study [11]. Instead, Van der Does and coworkers studied early onset schizophrenic patients and extracted three dimensions: positive, negative, and disorganized dimensions [6] The only study which assessed BPRS-E factor structure in an acute setting was performed by Ventura et al. [2], who described four components (depression/anxiety, manicexcitement, negative symptoms, positive symptoms) in a sample of schizophrenic and bipolar patients, assessed during an acute psychotic re-exacerbation.

In our analysis, the symptom "thought disturbance" was included in the component which was mainly characterized by uncooperativeness, suspiciousness, and hostility, thus labelled "Positive symptoms" cluster. Unexpectedly, hallucinations did not loaded on this component. This may be due to a dichotomised nature of the hallucination symptom, which may be only either present or absent, thus reducing the likelihood of a good correlation with other positive symptoms.

As far as the main aim of the study was concerned, we studied the relationship between symptom dimensions and measures of short-term outcome such as the severity of symptoms at discharge, the duration of acute hospitalization, and the presence of aggressive behaviours during acute hospitalization.

Our regression analysis showed that the excitement/activation component had the highest weight in predicting the persistence of psychopathological symptoms at discharge and the occurrence of aggressive behaviours, particularly verbal aggression and aggression against others. The relationship between excitement symptoms and aggression is consistent with a recent study which showed that a diagnosis of mania, or presence of manic symptoms such as heightened subjective functioning, expansive mood, or hyperactivity, independently predicted aggression in psychiatric patients at onset of their diseases [22]. This has also some precedence in symptoms of excitement predicting aggressive behaviours found in acute-onset disorders in studies by Milton et al. [23] and Volavka et al. [24].

The severity of symptoms at discharge was also predicted by the negative symptoms component at presentation, which was also associated to longer duration of hospitalization. This is consistent with the idea that negative symptoms are associated to a poorer response to short-term treatment. However, in the present study the positive symptoms component had the highest weight in predicting the duration of acute hospitalization, and had a moderate contribute in predicting verbal aggressive behaviours.

Other studies performed similar analyses and found results comparable to our findings $[17,18,27,28]$ : Hopko and coworkers [28] found that the resistance dimension, as well as positive symptoms, were helpful in predicting the length of stay in a sample of patients acutely admitted to a general psychiatry facility. Anderson et al. [17], however, showed that negative symptoms factors were the best predictor of the 
length of stay. The discrepancies between these studies might be due to the fact that the sample of Anderson was constituted by patients affected by severe and persistent illness in a medium-term care setting (mean stay more than 100 days). Since in such sample negative symptoms tend to be more treatment resistant than other symptoms, it is conceivable that in Anderson's study negative symptoms may be the best correlate of the length of hospitalization. In our study, based on emergency facility setting, we found both positive and negative symptoms to predict prolonged acute hospitalizations. Although the "Positive symptoms" dimension had the strongest association with extended hospitalisation, it was not associated to more severe psychopathology at discharge. A possible explanation of this apparent contradiction is that hostility, suspiciousness, and uncooperativeness often represent an obstacle to therapeutic alliance between psychiatrists and patients and lead to overestimate the severity of illness and delay the discharge of patients showing such symptoms.

Finally, the depressive/anxiety component was associated only to self-aggressive behaviours, and did not predict any other indicator of poor outcome. This is consistent with a recent study by Jager et al. [29], which observed that depressive symptoms predicted a favourable outcome but most of the effect was due to the influence of positive and negative symptoms at admission. In a previous study, affective symptoms in an early phase of schizophrenia among resulted predictors of a good outcome. Instead, depressive symptoms predicted a poor outcome in the latest stage of the illness and in chronic patients [30].

This study has several limitations, including the use of indirect measures of poor outcome, such as persistence of symptoms at discharge and length of hospitalization. Application of principles of clinical significance could have been desirable. Moreover, the only variables taken into consideration here as predictors of outcome are symptom dimensions, although other clinical and socio-demographic characteristics are well known to play an important role.

In conclusion, we have found dimensions of symptoms of acute re-exacerbating psychosis to differentially predict distinct measures of poor clinical outcome. The main finding is that the symptom dimension of excitement and activation had the strongest association with acts of aggression and severity of psychopathological symptoms at discharge. Positive and negative symptoms dimensions, also, predicted measures of poor outcome.

\section{ACKNOWLEDGEMENTS}

The authors acknowledge the patients included in the study. The authors would like also to thank Dr. Paolo M. Boato for his helpful suggestions.

Dr. Colasanti would like to acknowledge the colleagues of the International College of Affective Neuroscience for their scientific support.

\section{CONFLICTS OF INTERESTS}

The authors disclose no conflict of interests. No financial support or compensation has been received from any individual or corporate entity for research or professional service and there are no personal financial holdings that could be perceived as constituting a potential conflict of interest.

\section{REFERENCES}

[1] Overall JE, Gorham DR. The brief psychiatric rating scale. Psychol Rep 1962; 10: 799-812.

[2] Ventura J, Nuechterlein KH, Subotnik KL, Gutkind D, Gilbert EA. Symptom dimension in recent-onset schizophrenia and mania: a principal component analysis of the 24- item Brief Psychiatric Rating Scale. Psychiatric Res 2000; 97(2-3): 129-35.

[3] Shafer A. meta-analysis of the psychiatric rating scale factor structure. Psychol Asses 2005; 17 (3): 324-35.

[4] Ruggeri M, Koeter M, Schene A, et al. Factor solution of the BPRS-expandedversion in schizophrenic outpatients living in five European countries. Schizophr Res 2005; 75 (1): 107-17.

[5] Hafkenscheid A. Psychometric evaluation of a standardized and expanded. Brief psychiatric rating scale. Acta Psychiatr Scand 1991; 84(3): 294-300.

[6] Van der Does AJ, Linszen DH, Dingemans PM, Nugter MA, Scholte WF. A dimensional and categtorical approach to the symptomatology of recent-onset schizophrenia. J Nerv Ment Dis 1993; 181(12): 744-9.

[7] Dingemans PMAJ, Linszen DH, Lenior ME, Smeets RM. Component structure of the expanded brief psychiatric rating scale. Psychopharmacol 1995; 122: 163-267.

[8] Burger GK, Calsyn RJ, Morse GA, Klinkenberg WD, Trusty ML. Factor structure of the expanded brief psychiatric rating scale. J Clin Psychol 1997; 53(5): 451-4.

[9] Long JD, Brekke JS. Longitudinal factor structure of the brief psychiatric rating scale in schizophrenia. Psychol Assess 1999; 11(4): 498-506.

[10] Ventura J, Lukoff D, Nuechterlein KH. Brief psychiatric rating scale expanded version scales, anchor points, and administration manual. Int J Methods Psychiatric Res 1993; (3): 227-43.

[11] Kopelovicz A, Ventura J, Liberman RP, Mintz J. Consistency of psychiatric rating scale factor structure across broad spectrum of schizophrenia patients. Psychopathology 2008; 41 (2): 77-84.

[12] Robinson DG, Woerner MG, Alvir JMJ, et al. Predictors of treatment response from the first episode of schizophrenia or schizoaffective disorder. Am J Psychiatry 1999; 156: 544-9.

[13] McEvoy JP, Schooler NR, Wilson WH. Predictors of therapeutic response to haloperidol in acute schizohrenia. Psychopharmacol Bull 1991; 27: 97-101.

[14] Hatta K, Nakamura H, Matsuzaki I. Acute-phase treatment in general hospitals: clinical psychopharmacologic evaluation in firstepisode schizophrenia patients. General HospPsychiatry 2003; 25: 39-43.

[15] Cuesta MJ, Peralta V, de Leon J. Schizophrenic syndromes associated with treatment response. Prog Neuro-Psychopharmacol Bio Psychiatry 1994; 18: 87-9.

[16] Crespo-Facorro B, Pelayo-Terán JM, Pérez-Iglesias R, et al. Predictors of acute treatment response in patients with a first episode of non-affective psychosis: sociodemographics, premorbid and clinical variables. J Psychiatr Res 2007; 41(8): 659-66.

[17] Anderson SW, Crist AJ, Payne N. Predicting inpatient length of stay with the expanded version of the brief psychiatric rating scale. Psychiatric Serv 2004; 55: 77-9.

[18] Biancospino B, Barbui C, Grassi L. The BPRS-E as predictor of length of stay in a residential facility. Psychiatric Serv 2004; 55(1): 77-9.

[19] Serper MR, Goldberg BR, Herman KG, et al. Predictors of aggression on the psychiatric inpatient service. Compr Psychiatry 2005 46(2): 121-7.

[20] Steinert T, Wolfle M, Gebhardt RP. Measurement of violence during in-patient treatment and association with psychopathology. Acta Psychiatr Scand 2000; 102(2): 107-12.

[21] Nolan KA, Volavka J, Czobor P, et al. Aggression and psychopathology in treatment-resistant inpatients with schizophrenia and schizoaffective disorder. J Psychiatr Res 2005; 39(1): 109-15.

[22] Dean K, Walsh E, Morgan C, et al. Aggressive behaviour at first contact with services: findings from the AESOP First Episode Psychosis Study. Psychol Med 2007; 37(4): 547-57.

[23] Milton J, Amin S, Singh SP, et al. Aggressive incidents in firstepisode psychosis. Br J Psychiatry 2001; 178: 433-40. 
[24] Volavka J, Laska E, Baker S, Meisner M, Czobor P, Krivelevich I. History of violent behaviour and schizophrenia in different cultures. Analyses based on the WHO study on Determinants of Outcome of Severe Mental Disorders. Br J Psychiatry 1997; 171: 9-14.

[25] Kay SR, Wolkenfeld F, Murrill LM. Profiles of aggression among psychiatric patients: I. Nature and Prevalence. J Nerv Ment Dis 1988; 176: 539-46.

[26] Arndt S, Turvey C, Andreasen NC. Correlating and predicting psychiatric symptom ratings: Spearman's $r$ versus Kendall's tau correlation. J Psychiatr Res 1999; 2: 97-104.
[27] Salokangas RKR. Symptom dimension and outcome in schizophrenia. World Psychiatry 2003; 2(3): 172-8.

[28] Hopko DR, Lachar D, Bailley SE. Assessing predictive factors for extended hospitalization at acute psychiatric admission. Psychiatric Serv 2001; 52: 1367-73.

[29] Jäger M, Riedel M, Schmauß M, Pfeiffer H, Laux G, Naber D. Depression during an acute episode of schizophrenia or schizophreniform disorder and its impact on treatment response. Psychiatry Res 2008; 158(3): 297-305.

[30] Salokangas RKR. Symptom dimension and outcome in schizophrenia. World Psychiatry 2003; 2(3): 172-8.

Received: October 15, 2009

Revised: May 31, 2010

Accepted: June 06, 2010

(C) Colasanti et al.; Licensee Bentham Open.

This is an open access article licensed under the terms of the Creative Commons Attribution Non-Commercial License (http://creativecommons.org/licenses/by-nc/3.0/) which permits unrestricted, non-commercial use, distribution and reproduction in any medium, provided the work is properly cited. 